\title{
Estrategias Pedagógicas mediadas por las TIC, para la Sana convivencia ${ }^{1}$
}

\section{Pedagogical Strategies Mediated by Tic, for Healthy Living}

DOI: http://dx.doi.org/10.17981/cultedusoc.9.3.2018.23

Artículo de investigación. Fecha de recepción: 15/06/2018. Fecha de aceptación: 27/11/2018

\author{
Leonor Surmay²; \\ Francisco Viloria; Laura Ospino; Esperanza Meriño; Glenia Mendoza; \\ Liliana Barrios; Javier Peña; Merlys Choperena; Ena Gámez; \\ Bertha Barraza; Adalgiza Barrios y Lilia Ortíz ${ }^{3}$ \\ IED Liceo Ariguaní, sede: Urbana de Niñas (Colombia) \\ leosur@gmail.com
}

Para citar este artículo:

Surmay, L., Viloria, F., Ospino, L., Meriño, E., Mendoza, G., Barrios, L., Peña, J., Choperena, M., Gámez, E., Barraza, B., Barrios, A. y Ortíz, L. (2018). Estrategias Pedagógicas mediadas por las TIC, para la Sana convivencia. Cultura. Educación y Sociedad 9(3), 201-208. DOI: http://dx.doi.org/10.17981/cultedusoc.9.3.2018.23

\section{Resumen}

El objetivo del presente estudio es identificar situaciones de cyberbulling en la Institución Educativa Liceo Ariguaní para desarrollar acciones que permitan a los niños, jóvenes y padres de familia, fortalecer las relaciones interpersonales y promover la sana convivencia. La investigación es de tipo cualitativa, de corte descriptivo, se empleó la entrevista estructurada como instrumento y aplicado a 40 estudiantes de los grados de 5 a 9 de la sede Urbana de Niñas. Como conclusiones se puede decir que es notoria la importancia de la familia, escuela y comunidad y qué papel juega estos tres pilares en la formación del ser humano, sin embargo, se deben intensificar las estrategias pedagógicas y la participación de los entes para promover ambientes educativos armónicos.

Palabras clave: Estrategias pedagógicas; TIC; Convivencia; Familia; escuela; sociedad.

\section{Abstract}

The objective of this study is to identify situations of cyberbullying at the Liceo Ariguaní Educational Institution to develop actions that allow children, youth and parents to strengthen interpersonal relationships and promote healthy coexistence. The research is qualitative, descriptive, structured interview was used as an instrument and applied to 40 students from grades 5 to 9 of the Urban Headquarters of Girls. conclusions can be said that the importance of the family, school and community and what role these three pillars play in the formation of the human being; however, pedagogic strategies and the participation of the entities to promote harmonious educational environments must be strengthened.

Keywords: Pedagogical strategies; TIC; Coexistence; Family; school; society.

\footnotetext{
1 Este artículo ha sido derivado del Programa de Fortalecimiento de la Cultura Ciudadana y Democrática CT+I a través de la IEP apoyada en TIC en el Departamento de Magdalena: CICLON.

2 Líder del grupo de investigación "Los pacificadores”.

3 Docentes pertenecientes al grupo de investigación "Los pacificadores” de la IED Liceo Ariguaní, sede: Urbana de Niñas. Ciclón Magdalena, estrategia $\mathrm{N}^{\circ} 2$. Universidad De la Costa CUC.
}

- The author; licensee Universidad de la Costa - CUC. 


\section{Introducción}

La sociedad actual demanda que las organizaciones reconozcan su capacidad de ocasionar serios impactos sobre el medio, en sus dimensiones social, natural y económica (Hernández \& Jiménez, 2015), la escuela no es algo que escape a estas demandas. Las escuelas reconocen, además las múltiples contribuciones que la sociedad les genera y por ello es apasionante ser protagonistas y contar con la oportunidad de contribuir de modo constructivo a la formación integral y participativa.

Teniendo en cuenta lo anterior es pertinente mencionar que en La Institución Educativa Departamental Liceo Ariguaní Sede Urbana de Niñas se evidencia un sin número de situaciones que desestabilizan el buen funcionamiento y la sana convivencia. Esta situación involucra tanto a estudiantes como docentes, y se agudiza cada día no contemplando mecanismos que permitan un manejo de las normas establecidas.

Aunque son diversas las razones por las cuales los estudiantes se agreden, las dificultades que se presentan en el aula de clase, dentro del plantel y fuera de él ponen en riesgo la estabilidad y la armonía en la institución educativa, ya que alteran la dinámica institucional y afecta el rendimiento académico de la población escolar, debilitándose las relaciones interpersonales, siendo esta una barrera para el trabajo en equipo y el manejo de las normas básicas del respeto ciudadano. Debido a estas debilidades se generan distintos resultados, entre ellos la protesta que, según Cruz. (2016) "ésta adquiere diversos sentidos, en algunos casos puede orientarse a rechazar determinados actos de violencia" (p. 42)

Desde todas las áreas del saber y sus componentes debemos facilitar a todos los estudiantes y comunidad educativa en general un ambiente de diálogo, comunicación y de interrelaciones colectivas para la sana convivencia de cada uno de ellos.
Como mediador común aparecen la colaboración y la cooperación como un producto de la participación y el resultado del contacto que tienen a diario cada uno de los estudiantes (Antolín, Oliva, Pertegal y López, 2012).

En efecto todas las áreas del saber son un medio de cooperación y de relación ciudadana que se convierte en una estrategia que facilita la inclusión del otro, además de promover aprendizajes referidos al trabajo en equipo y colaborativo generando la aceptación de las normas, la resolución de problemas y conflictos entre otros.

La relación entre conceptos y convivencia ciudadana la determinan los niveles de significación aplicados a la formación cotidiana en contextos culturales preestablecidos.

Sin duda las áreas de la educación buscan la comprensión de admitir al otro como es, ya que con esto se busca disminuir las expresiones de agresividad en la práctica de ambiente sano, promoviendo los valores ciudadanos y las manifestaciones de afecto y el acatamiento de las normas que nos sirven para el fortalecimiento de los logros de objetivos colectivos para trazar metas que superen los mismos desafíos u obstáculos para una mejor convivencia en la Sede Urbana de Niñas.

En este sentido, la escuela es el escenario propicio donde se alberga el individuo desde la niñez, convirtiéndose en uno de los principales espacios de intervención para reducir los comportamientos de intolerancia y agresividad de los niños y de esta institución.

La educación permite procesos de autoconocimiento, interacción, socialización e inclusión, es por ello que a la institución educativa le corresponde desde su propuesta formativa, inculcar este tipo de experiencias en la realización de tareas escolares y asegurar en los estudiantes un futuro lleno de goce, honestidad, respeto, responsabilidad, tolerancia y colaboración consigo mismo y con los demás. 
Es pertinente mencionar que a través de la observación realizada en la I.E.D Liceo Ariguaní, sede Urbana de Niñas se logró evidenciar la importancia de implementar estrategias que ayuden a mejorar el clima escolar por cuanto se ha evidenciado dificultad para lograr este fin. Fortalecer valores fundamentales que conlleve al sujeto enfrentar diferentes situaciones adversas sin hacer uso de la violencia en cualquiera de sus formas, debido a que en los recesos escolares y en horas libres existe un alto índice de situaciones conflictivas que no se resuelven de manera adecuada.

Por tanto, en esta investigación se plantea el desarrollo de un ambiente saludable, permitiendo detectar las situaciones de ciberbullying y así poder generar estrategias que den solución a la problemática evidenciada, participando en la modernización de la educación con la utilización de tecnologías de la información y la comunicación, la transformación de los procesos de enseñanza-aprendizaje; enfatizando en la prevención del cyberbullying.

Es importante resaltar que todas las actividades que se realicen en función de mitigar cualquier tipo de problemática debe partir del interés colectivo, pues tal como lo expone Herrera (2014), citado en la Corte Constitucional de 1991 "el interés colectivo es definido como un interés que nos atañe a todos y cada uno de los miembros de una colectividad determinada".

Es a partir de la implementación de ambientes saludables que se pretende llevar a cabo un proceso de fortalecimiento de diferentes valores con los estudiantes. El ambiente está dispuesto de modo que los estudiantes puedan interactuar en el colegio para observar el comportamiento de los mismos ante la propuesta realizada, razón por la que se determinó realizar una serie de actividades de índole lúdico-recreativo y académico haciendo uso del tic; en los cuales se ha de abordar el fortalecimiento del estándar de convivencia y paz.
Siendo ésta una investigación que contribuye al mejoramiento de la convivencia escolar, se recurre al abordaje de temáticas acordes, mediante el uso de recursos, instrumentos y materiales digitales educativos para que se constituyan en un apoyo importante para fortalecer cambios culturales significativos en los estudiantes, atendiendo al acuerdo de normas y mínimos éticos para la convivencia, reconocimiento y uso por parte de los mismos (Samper \& Maussa, 2014), a fin de mejorar la convivencia y la paz en el aula, en la sede y por tanto en la familia y comunidad.

El Ministerio de Educación Nacional con el ánimo de contribuir a la formación de ciudadanos activos que aporten a la construcción de una sociedad democrática, participativa, pluralista e intercultural, trabaja en la puesta en práctica del decreto 1965 de septiembre 11 de 2013, que el presidente de la República Juan Manuel Santos sancionó, "Por el cual se reglamenta la Ley 1620 de 2013", que crea el "Sistema Nacional de Convivencia Escolar y Formación para el Ejercicio de los Derechos Humanos, la Educación para la Sexualidad y la Prevención y Mitigación de la Violencia Escolar" (Ministerio, 2013).

Según (Ministerio, 2013). La ley 1620 de 2013 "crea una ruta de atención en casos de violencia y un sistema nacional único de información para reportar estos casos de violencia y de embarazo en adolescentes, y da la posibilidad de brindar incentivos a quienes cumplan las exigencias y expectativas de la convivencia, así como imponer sanciones a quienes no lo hagan" p.p 23.

Cuando se habla de violencia, resaltamos que no es nada novedoso, desde que existen las relaciones, existe la violencia, lo que ha cambiado con el pasar del tiempo es es la manera en la que se manifiesta, en donde se originan diversas formas de apoyándose de los avances de la ciencia. Es la violencia la que ha alcanzado todos los ámbitos de participación (escuela, familia, iglesia, política,). 
Es trascendental mencionar que una buena convivencia exige empatía cognitiva y emocional; por lo que es necesaria cierta reciprocidad de comportamiento tolerante y solidario de aquellos que conviven. Si no se diera esa reciprocidad de sentimientos positivos entre los que viven en sociedad, se podría generar un conflicto de convivencia.

La escuela se convirte en el camino con el que cuentan las sociedades cuentan para propiciar entornos de sana convivencia y transformación de ciertos comportamientos, ademasd e equiparse de herramientas para el afrontamiento del bullyng y el cyber-bullying.

En este orden de ideas y dada la mala convivencia que prevalece en la IED Liceo Ariguaní sede 3, Urbana de Niñas a través de manifestaciones como agresividad y faltas de respeto que tiene a los estudiantes bajo estrés permanente y un ambiente lleno de expectativas negativas en lo referente a ¿cuándo va a ser el próximo enfrentamiento?, o si estaba haciendo una broma en apariencia inocente a un compañero ¿por qué él o ella me responde con un golpe o con un insulto? Estas y otros interrogantes de la misma línea son a diario manifestados por los estudiantes quienes a través de este proyecto ven la oportunidad para aprender herramientas y estrategias para mejorar su interactuar con sus pares.

Por otra parte, es el internet quien se ha convertido en uno de los medios de comunicación favoritos por la población adolescente, los cuales han sido participe de todos estos avances, haciendo parte de la actual era digital. Dicha era ha servido para que dentro de su multiplicidad de ofertas por aprender, los agresores adquieran nuevas formas de generar violencia, haciendo una especie de catarsis de las frustraciones y dificultades que encuentra en su entorno dando lugar a un nuevo tipo de bullying:
Entender la educación como una herramienta al servicio de la convivencia democrática ha pasado de ser una máxima pedagógica aceptada de forma mayoritaria, para convertirse en un reto educativo de primer orden. Convivir no es sólo vivir en el mismo lugar sino en compañía y comunión con otros. Es un gran desafío lograr la convivencia cuando sabemos que tienden a primar los intereses individuales y las opiniones son tan diversas (Guerrero y Cepeda 2016).

\section{Convivencia Escolar}

No cabe duda que dada la pluralidad y multiculturalidad que se vive en el mundo actual, como es de conocimiento general globalizado, la convivencia se constituye como un eje fundamental en el desarrollo de la educación. Tal como lo explican Rosario Del Rey, Rosario Ortega, Irene Feria en la Revista Iberoamericana de Educación, al citar Ortega y Martin (2010).

La convivencia surge como una necesidad prevalente de los individuos al interaccionar, y es en el escenario educativo en donde toma gran fuerza las distintas situaciones que de alguna u otra forma debilitan los lazos armoniosos que se construyen, y es aquí donde se fomenta la práctica de normas y pautas de comportamientos esperados, que propicien el bien común. (Del Rey, 2009.)

En este orden de ideas la convivencia más que aprenderla en la escuela como un aspecto más del curriculum, hay que construirla como factor esencial desde la formación escolar. En concordancia con lo expuesto en el IV Congreso Profesorado y Convivencia (MEC, 2008c).

"La convivencia, por su propia y compleja naturaleza de red de redes de relaciones, es frágil y puede ser agredida desde distintas instancias, personas y grupos de personas. Tampoco implica la ingenuidad de que las cosas pueden ir bien en la convivencia si no se ocupan de ella los agentes educativos" (pp. 22). 
La educación es un proceso dinámico, perfectivo, intencionado, dialógico, que orienta la personalización y la socialización del sujeto humano. Esta educación está basada en los principios como: individualidad, naturalismo, unidad, libertad y autonomía, cooperación actividad e interés. En todo este proceso definir la convivencia como el escenario donde interactúan los integrantes de la comunidad educativa es trascendental.

\section{Comunidad Educativa}

Tal como lo expone Eduardo Espino López, en su artículo de opinión importancia de la comunidad educativa:

La comunidad educativa es el conglomerado de actores de primer orden involucrados directamente en el proceso de formación de niños y jóvenes; es más que las instalaciones escolares y los currículos formales de contenidos académicos. Está conformada por los estudiantes, los docentes, el personal administrativo, os reglamentos y los programas didácticos en constante interacción. En ese ámbito el proceso de enseñanza aprendizaje se da de manera continua, no solo en el aspecto formal, sino en las interrelaciones entre cada uno de los actores. Son comunidades en la que existe el compromiso de formar a futuros ciudadanos, productivos y responsables (Espino, 2014).

Sin duda la educación tiene el potencial de lograr que la democracia en su sentido original y amplio se afiance en la base cultural de la sociedad, es decir, en la escuela, potencial que conlleva la necesidad de una convivencia proactiva en el seno de toda la comunidad educativa. Gracias a las TIC, las películas, los videos, la música, los videojuegos, los amigos, las noticias, el conocimiento y el mundo entero, están a un clic de distancia.

Mora- Merchán, (s.f) Pone de manifiesto una situación real como es la llegada y de la tecnología, específicamente de la tec- nología móvil al entorno escolar. Destaca este estudio algunos de los motivos de este boom en la comunidad escolar como son que la universalización de los dispositivos electrónicos, es decir, cada día es más fácil acceder o adquirir uno de estos artefactos, de hecho, las escuelas públicas tienen a disposición para los estudiantes tabletas con servicio de internet.

Otro factor es el desarrollo de entornos virtuales diseñados para el establecimiento de relaciones afectivas, tal es el caso de la red social Facebook en donde a diario interactúan millones de personas incluso sin conocerse cara a cara.

También resalta la trasposición de la dinámica de las relaciones de lo físico a lo virtual, es decir, que si bien es cierto en la mayoría de los casos los estudiantes se llevan bien entre sí, también lo es el hecho de que las malas relaciones cambian de escenario y lo virtual se hace "idóneo" para expresar violencia y otro tipo de manifestaciones de esta índole.

Ahora, nos asalta la duda, ¿es el Cyberbullying una nueva manera de acosar y de agredir o simplemente es la evolución de la forma tradicional de hacerlo?, para este grupo de investigación es sin lugar a dudas la segunda opción, la tecnología ha influido en todos los ámbitos de la vida de las personas y de manera contundente en la de los jóvenes y adolescentes que han ampliado el espectro de sus relaciones a lo virtual de tal modo que trasladan sus relaciones positivas y negativas a este entorno teniendo en cuenta las ventajas que la virtualidad ofrece.

\section{Metodología}

La investigación esta soportada en la mirada cuantitativa, pues es esta quien brinda herramientas para el estudio de los fenómenos, cuantificarlos y generar resultados desde el contexto en donde interactúan los sujetos. 


\section{Población y muestra}

Los participantes son los estudiantes de $5^{\circ}$ a $9^{\circ}$ de la Institución educativa Liceo Ariguaní, sede Urbana de Niñas. Se tomó una muestra de (40) estudiantes.

Técnicas e instrumentos de recolección de la información

Dado el tipo de investigación cuantitativa y las características del escenario de investigación se decide utilizar como técnicas de recolección una encuesta de esta manera la información es clasificada y tabulada dando resultados estadísticos referentes a cada pregunta.

\section{Procedimiento}

Se construyeron los contenidos de acuerdo de acuerdo a la problemática estudiada. La escala aplicada fue enviada a pares expertos para su respectiva validación. Se hizo una prueba piloto, tomando los aportes más significativos de los aspectos evaluados.

Culminada la prueba piloto se envió de nuevo a los jueces expertos, quienes hicieron las correcciones y recomendaciones pertinentes. Finalmente se procede a realizar la aplicación a la población objeto de estudio.

El diseño de las preguntas, tuvo una guía que permitiera elaboración definitiva del cuestionario, basándose en propósitos específicos de la investigación, se realizó una validación presentada a expertos y fue aplicada a estudiantes de $5^{\circ}$ a $9^{\circ}$ grado de la IED Liceo Ariguaní.

\section{Resultados}

En este apartado de la investigación se dará relevancia a los hallazgos encontrados.

Primera pregunta: ¿Qué es el Cyberbullying?
El 57.5\% de los entrevistados si sabe que es el Cyberbullying, 17.5 de los entrevistados dio una definición con leves conocimientos, y un $26 \%$ no conoce del concepto.

Con relación a la segunda pregunta: ¿Ha sido víctima de Cyberbullying?

El 55\% de los entrevistados manifiesta que sí, seguido de un $10 \%$ que afirma que algunas veces y finalmente un $80 \%$ que manifiesta no haber sido víctima de este tipo de acoso.

La tercera pregunta: ¿Consideras que los valores que pueden ayudar a mantener una sana convivencia entre los miembros de la comunidad educativa urbana de niñas?

En este ítem el 100\% de los encuestados consideraron que los valores juegan un papel primordial en la convivencia, es decir, para ellos es muy importante cultivar respeto, amistad, tolerancia y amor para poder llevar relaciones armónicas y saludables.

La cuarta pregunta: ¿Cree usted que el diálogo es un medio por el cual los niños, jóvenes, padres de familia, pueden tomar conciencia sobre la importancia de modificar algunos comportamientos asegurando mejores relaciones?

El 100\% de los participantes consideran que la mejor opción para ayudar a los niños y jóvenes a modificar comportamientos negativos al momento de interactuar con sus pares es el dialogo en familia.

\section{Conclusiones}

Los resultados permiten mostrar que tanto la familia, los docentes y estudiantes conforman un sistema que debe fortalecer valores, intensificar la armonía y sobre todo buscar herramientas que mitiguen todo tipo de anomalías que entorpezcan las relaciones interpersonales.

Con la llegada de las nuevas tecnologías, los estudiantes han tenido la oportunidad de adquirir nuevas destrezas y a su vez han 
diversificado los aprendizajes, sin embargo estas herramientas también han tenido sus desventaja en cuanto a las relaciones sociales, tal es el ejemplo al interior de las instituciones educativas con la presencia del cyberbullying, que si bien es bajo el número de casos presentados en la IED Liceo Ariguaní, tanto los docentes como padres de familia deben estar preparados para saber cómo mitigar este flagelo que puede aumentar en la medida en que los estudiantes tengan mayores acceso a la red, al igual que se debe reorientar las practicas con los estudiantes, como lo expresa Gutiérrez (2014) la conveniencia pacifica deberá, en todo caso, estar sujeta al cumplimiento de metas concretas conforme al alcance y resultados en los procesos.

Es notoria la importancia de la familia, escuela y la sociedad en la formación de los individuos. La familia por su parte es la primera escuela, en donde se sientan las bases de formación en valores, la escuela reafirma las competencias del saber, y extiende la puesta en práctica lo que la familia oferta, y finalmente la sociedad contribuye o debilita el accionar de sus integrantes.

No cabe duda de que realmente se puede evidenciar dentro y fuera del aula que los estudiantes están demandando el cambio en las prácticas de aula, pues si la tecnología está presente en la vida de los seres humanos porque no estar presente en el aula de clases, diseñar estrategias mediadas por las Tic favorece crear un ambiente agradable, donde prime el respeto por las diferencias que tanto conflicto causa en las escuelas.

\section{Referencias}

Antolín, L., Oliva, A., Pertegal, M., \& López, A. (2012). Desarrollo y validación de una escala de valores para el desarrollo positivo adolescente. Psicothema (en línea), 153-159.
Avendaño, I., Cortés, O. y Guerrero, H. (2015) Competencias sociales y tecnologías de la información y la comunicación como factores asociados al desempeño en estudiantes de básica primaria con experiencia de desplazamiento forzado Diversitas: Perspectivas en Psicología, 11(1). 13-36. Recuperado de http://www. scielo.org.co/pdf/dpp/v11n1/v11n1a02. pdf.

Berra, M. y Fernandez, R. (2017). Convivencia escolar y habilidades sociales. Recuperado de https://educrea.cl/convivencia-escolar-y-habilidades-sociales/

Cruz, E. (2016). El ciclo de protesta 20102016 en Colombia. Una explicación. Jurídicas CUC, 12(1), 31-62. http://dx.doi. org/10.17981/juridcuc.12.1.2016.3.

Del rey, R. (2009). Convivencia Escolar: fortaleza de la comunidad educativa y protección ante la conflictividad escolar. ISSN 02 Revista Interuniversitaria de Formación del Profesorado, 159-180.

Elipe, P., Ortega, R., Hunter, S. y Del Rey, R. (2012). Inteligencia emocional percibida e implicación en diversos tipos de acoso escolar. Psicología Conductual, 169-181.

Espino, E. (2014). La Prensa. Recuperado de: https://impresa.prensa.com/opinion/ importancia-educativa-Eduardo-Espino-Lopez_0_3852364804.html

García L. y Lopez, R. (2012). Convivir en la escuela. Una propuesta para su aprendizaje por competencias. Revista de Educación. 531-555. Recuperado de: https://www.mecd.gob.es/dctm/revistade-educacion/articulosre356/re35622. pdf?documentId=0901e72b81203172

García, C. (2012). Familia y convivencia escolar. [Tesis de Maestría]. Facultad de Educación. Universidad de Almería. España. Recuperado de http://repositorio. ual.es/bitstream/handle/10835/1178/ Garcia_Escamilla_Carmen_Maria. pdf?sequence $=1$ 
Giménez, A., Maquilón, J. y Arnaiz P. (2014). Acceso a las tecnologías, rendimiento académico y cyberbullying en escolares de secundaria. Revista Iberoamericana de Psicología y Salud, 119-133.

Gutiérrez, J. (2014). Costos sociales de transacción de la ley de restitución de tierras en Colombia: un país sin reforma agraria. Juridicas CUC, 10(1), 157196.

Hernández, H. y Jiménez, A. (2015). Responsabilidad social empresarial, zona urbana del distrito de Barranquilla: estudio etnográfico. Económicas CUC, $36(2), 9-18$.

Herrera-Tapia, B. (2014). Las acciones colectivas en Colombia frente a una realidad global: El derecho de consumo. Justicia, 25. 70-81.

Millán, T. (2012). Investigación cualitativa. Universidad de las Américas. Recuperado de. https://metodoinvestigacion. wordpress.com/2008/02/29/investigacion-cualitativa/

Mora, J. (2015). Cyberbullying: un nuevo reto para la convivencia en nuestras escuelas. Información psicológica, vol. 3. (94).60-70.
Moreno, D. (2009). Relación entre el clima familiar y el clima escolar: el rol de la empatía, la actitud hacia la autoridad y la conducta violenta en la adolescencia. Red de Revistas Científicas de América y El Caribe, 123 -136.

Muñoz, M., Lucero, B., Cornejo, C., Muñoz, P. y Araya, N. (2014). Convivencia y clima escolar en una comunidad educativa inclusiva de la provincia de Talca, Chile. Revista electrónica de investigación educativa, 16(2). 32.

Mursi, S. (2012). Acoso escolar y estrategia de prevención en educación Básica escolar y nivel medio. [Tesis de Pregrado]. Universidad Tecnológica intercontinental. San Lorenzo, Paraguay.

República de Colombia. Min Tic. (2017). En Tic Confío. Recuperado de http://www. enticconfio.gov.co/que-son-las-tic-significado

República de Colombia. Ministerio de Educación. (15 de marzo de 2013). Ley de convivencia escolar. Diario Oficial No. 48.733. Recuperado de http://www.mineducacion.gov.co/1759/w 3 -article-322486.html

Samper, J. y Maussa, E. (2014). Desarrollo moral y competencias ciudadanas en la juventud universitaria. Juridicas CUC, 10(1), 43-60. 